\title{
Deceased Donor Kidney Allocation Schemes and International Exchange
}

Uwe Heemann1, Rainer Oberbauer2, Ben Sprangers3, Cenk Gökalp4, Frederike Bemelman5

1 Department of Nephrology, Klinikum rechts der Isar, Technische Universität München, Munich, Germany

2 Division of Nephrology and Dialysis, Department of Medicine III, Medical University of Vienna, Vienna, Austria

3 Department of Microbiology and Immunology, laboratory of molecular immunology (Regan Institute), KU Leuven and Division of nephrology, University Hospitals of Leuven, Both in Leuven Belgium

4 Department of Nephrology, Faculty of Medicine, Trakya University, Edirne, Turkey

5 Department of Nephrology, Division of internal Medicine, Amsterdam University Medical Center, Amsterdam, The Netherlands

Corresponding Author:

Prof. Dr. Uwe Heemann

Dept. of Nephrology

Klinikum rechts der Isar

Technische Universität München

Ismaningerstr. 22

81675 Munich

Germany

Tel.: +498941402231

Fax: +49 8941407734

E-Mail: uwe.heemann@mri.tum.de 


\begin{abstract}
Purpose of the review: In this review, we summarize different allocation schemes around the world and identify ways to exchange organs between countries.

Recent findings: The primary goal of transplantation is prolongation of patient survival and an improved quality of life. Most allocation systems try to match the potential survival of the organ with the life expectancy of its recipient.Kidney transplantation enables cost reductions by the avoidance of dialysis for the health care system, which is sometimes the driving force for state interventions to enhance donor recruitment

The major benefit from international exchange is the possibility to transplant highly sensitized patients or patients with rare HLA allele.

In a broad international exchange system, there are three possibilities: exchange of surplus organs, exchange of organs to support patients with a potentially excessive waiting time due to HLA antibodies or rare alleles, and exchange of organs to make the best possible match between donor and recipient.

Summary: It is possible to shape a hierarchical allocation scheme, which could be applicable in the majority of populations despite different geographical and socio-economic conditions: 1. Allocation to recipients with special requirements (High-Urgency, highly sensitized), 2. Identification of a within the region/country, 3 . In case no suitable recipient can be identified, offer countrywide or between countries, 4. For every organ transferred to another country, there must be an obligation to give one back.
\end{abstract}

Keywords: Allocation, kidney transplantation, waiting list, ischemia, age 


\section{Introduction}

Kidney transplantation is the best option for the treatment of end stage kidney disease regarding patient survival, quality of life and costs $(1,2)$. Increased demand for organs and inadequacy of appropriate supply require effective and fair rules of allocation. Although the problems are very similar around the world, allocation rules differ markedly between countries. Some allocation systems allocate regionally, some nationally and a very few across borders.

In addition to broadly accepted factors that affect graft and patient survival, local medical and social needs are taken into account in the development of allocation systems (Table 1 ) $(3,4)$.

Most allocation systems try to match organ and recipient survival, some focus on urgency or equal chance to receive an organ. Additionally, kidney transplantation enables cost reductions by the avoidance of dialysis, which is sometimes the driving force for state interventions in donor recruitment $(5,6)$.

This article gives an overview of current allocation systems for post-mortem donation and the thoughts behind them with the question: is there an integrated system that could work in most countries and enable organ sharing between countries?

\section{Factors which influence kidney allocation}

Kidney allocation is influenced by a number of considerations. Different allocation systems attribute different weight to social, biological and medical factors. Nonetheless, these factors dictate allocation rules and make it very complicated to develop rules that are applicable "worldwide".

Donor rate and number of patients on the waiting list

For obvious reasons donor rate is the most important factor of all. On the other hand, given a low number of patients on the waiting list, a mediocre donor rate may have the same effects as a high donor rate in a country with many patients on the waiting list. Thus, the relation between donors and patients on the waiting list is a critical issue.

Access to the waiting list

Access to the waiting list varies dramatically among the countries. As patients must have access to dialysis, the number of dialysis places in relation to the number of patients with CKD is essential. Some countries have no money for a sufficient number of dialysis units, and as a result many patients will die before they enter the waiting list. Some countries evaluate quality life years gained in order to grant access to dialysis (7); if a patient is considered to have no quality benefit by dialysis, dialysis is not initiated. In other countries, this is considered unethical so that even patients with serious dementia are put to dialysis.

There are also aspects of quality of dialysis treatment. Survival on dialysis differs markedly among countries (8). Again, there are multiple causes involved. If access for elderly patients is not regularly granted or patients die from other causes before dialysis can be started, average age on dialysis is lower than in systems of open and easy access to dialysis. Therefore, younger patients are more likely to live long enough so that they can be 
transplanted. Interestingly, Japan has the longest survival on dialysis and the highest average age on dialysis (8). Thus, cultural factors, such as adherence to treatment, genetic background as well as nutritional habits may contribute as much as the skill and philosophy of treating physicians.

\section{Waiting Time}

Waiting time is important for both medical and ethical reasons and is part of almost all allocation algorithm (4). However, it's impact seems to vary among the countries. Every year of additional waiting time reduces graft survival between $2 \%$ (USA) $(9,10)$ and $8-16 \%$ (Australia, New Zealand) (11). Furthermore, an increased waiting time has been associated with more frequent episodes of early rejections, severe vascular and/or antibody mediated rejections, graft failure, as well as death with functioning graft (DwFG). $(12,13)$.

Waiting time can be weighed differently in the algorithms for different populations. In Eurotransplant (ET) for example, HLA in pediatric recipients has a higher impact than in the regular program, and pediatric recipients get a 100 point bonus on listing and earn more points per HLA-match, whereas elderly have a separate program. The disadvantage of such a system is that the likelihood of a good HLA match is diminished due to a lower number of available potential donors for every individual patient category.

\section{Ischemia}

Warm and cold ischemia (CIT) are associated with delayed graft function $(14,15,16)$. While the effects of a CIT $<18 \mathrm{~h}$ are most likely marginal, at least in donation after brain death donors, $\mathrm{CIT}$ is the argument for local allocation in many countries. CIT may be more relevant for the allocation of DCD (donor after cardiac death) kidneys: proximity of the donor to the recipient center may be used as a prerequisite for allocation. Whether or not machine perfusion can help to ameliorate the effects of cold ischemic time is still a matter of debate. If logistics necessitate a time of $>18 \mathrm{~h}$ between induction of ischemia and start of warm perfusion, allocation has to be performed differently.

As it can be faster to ship an organ between continents rather than between small towns in neighbouring countries. Furthermore, the transport of the patient to the transplant center may take many hours. Current allocation schemes do not include these transport times.

\section{Age}

It is an ethical and emotional question whether children should be preferred over adults. Paediatric and young patients are prioritised in some allocation schemes with the result that middle-aged patients wait longer. In some countries, waiting times differ by weeks or months, in others by years. Thus, the shortening of waiting time in children, which compete for the same organs as adults, increases the number of deaths on the waiting list or, due to worsened comorbidities, the number of de-listings.

The term "Elderly" is most commonly attributed to patients over 60-65 years. In the developed countries, the population aged more than 65 years is increasing faster than the one aged less than 65 and so is the distribution of patients with end stage renal disease. 
In the US mortality was 4-6 times increased in recipients $>65$ years compared to recipients $18-<35$ years and death censored graft failure was $20-37 \%$ lower than in the younger age group $(17,18)$. However, transplantation is still associated with better survival compared to dialysis in any age group (19).

The subsequent evolution of the longevity matching principle in the US is well summarised in a recent review (4).

Various allocation schemes have tried to utilize kidneys from elderly donors rather than discard them $\left(20,21^{* *}\right)$. It might be worth mentioning that in the US only $5 \%$ of the donors are above 65yrs whereas in EU countries such as France, Germany, Italy and Spain they represent more than $25 \%$ of donors $(22 * *, 23)$.

In the UK and other countries recipient and donor age are matched, but the number of elderly recipients on the list is comparatively very low. A similar program would not work in countries with a disproportional distribution of elderly donors and elderly recipients on the waiting list.

\section{HLA Match}

The importance of an HLA match varies. While HLA class II is widely regarded to be important for acute rejection, the primary importance for HLA class I is most likely the presensitisation as well as long-time graft function $\left(24,25,26,27,28^{*}\right)$. Thus, all patients would profit from a HLA class II match, but only those who are young or fit enough to be likely retransplanted would benefit from a HLA class I match.

Nonetheless, only few countries take the difference between class I and II into consideration.

In an ideal world or a situation of donor abundance, the number of mismatches should be minimized particularly in younger recipients in order to decrease sensitisation and enhance the chances for retransplantations. However, nowadays waiting for a perfect match, especially in paediatric patients, may result in excessive waiting times.

Some allocation schemes such as in the UK therefore evaluate HLA mismatches in combination with recipient age to prolong graft survival and decrease the number of retransplantations. In this scheme HLA matches have more points in younger recipients than in older recipients (29). In most schemes, HLA mismatches are counted per se and are not weighed according to age.

\section{PRA}

The presence of anti-HLA antibodies increases waiting time and decreases graft survival (30).

If the potential recipient has antibodies against many HLA`s, the number of potential donors is reduced, which results in an increased waiting time. Some countries/organisations have developed schemes to counter this effect. One of the most effective and oldest is the "Acceptable mismatch program" introduced by Eurotransplant (31). In this program, a certain PRA level is required to enlist the patient. Once a donor is identified based on a virtual negative cross-match, the patient is prioritised. Graft and patient survival in this program are excellent $(32 *)$. One of the problems is, however, that some patients get offers 
immediately after enlisting, as the number of donors that fit the profile is very high. Thus, in the future the program within Eurotransplant will be based on donor frequency rather than the level of PRA alone.

Such a program is only feasible if the donor pool is large enough. Therefore, countries have to collaborate in order to find organs for patients with rare HLA alleles or very specific HLA antibodies such as has been tried in EUROSTAM (A Europe-wide Strategy to enhance Transplantation of highly sensitized patients on basis of Acceptable HLA Mismatches) $(33,34)$.

An international collaboration in Europe taking advantage of the fact that the transplantation antigens differ between European populations will be beneficial for this patient group. EUROSTAM simulation studies clearly showed that collaboration between Eurotransplant and transplant organizations in the UK, Spain, Greece, and the Czech Republic would lead to the identification of a suitable donor for about $25-30 \%$ of the 700 highly sensitized patients who at the moment do not have any chance to be transplanted within their own organization (38, manuscript in preparation).

\section{Combined Transplantation}

End stage renal disease is common before and after heart, lung, or liver transplantation. There are still some questions about benefit and outcomes for combined renal transplantation. Although some studies indicate a benefit of combined transplantation for the recipient, and most countries give combined transplantation priority over recipients for a single kidney, recent studies doubt the benefit particularly in subgroups $(35,36)$.

The downside of this prioritisation is that good kidneys are allocated to recipients for a combined transplantation with an enhanced risk for DwfG. Particularly in countries with a low donor rate in young adults, this has a remarkable impact on the waiting time of single kidney recipients although it is evident that transplantation prolongs life in this entity as well. Thus, such this policy is questioned or revoked in some countries.

\section{Renal Urgency}

Situations in which mortality is significantly increased if renal transplantation cannot be performed are considered to be renal urgency and constitute $0.02-3,1 \%$ of post-mortem donor kidney only transplantations $\left(37,38,39^{*}\right)$. Factors such as recipient age, HLA mismatch, waiting time and donor quality are ignored in renal urgency situations and urgency transplantations decrease the chance of non-urgent patients. Similar to other elements of allocation schemes, the definition of renal urgency differs among the countries. For example in the UK renal urgency is only defined for pediatric patients whereas in Brazil and Turkey it is defined for all haemodialysis and peritoneal dialysis patients that have access problems without age considerations (40). In Eurotransplant the definition is more complex. Currently patients with lack of access for dialysis, severe uremic polyneuropathy and severe bladder problems due to renal failure after simultaneous kidney and pancreas transplantation are accepted reasons (41).

An analysis of the renal urgency program in Eurotransplant gave the median waiting time for urgency transplantation as 5 days whereby more than $75 \%$ of patients were younger than 
55 years (41). On the downside, patients in an urgency program had more HLA mismatches albeit a similar graft survival, more retransplantations, and more frequent DwFG.

Given this situation, it is no wonder that the criteria for the enlisting in urgency programs vary between the countries. Some countries even have no urgency program at all.

\section{Is there a possibility for international organ exchange in the presence of different} allocation schemes within the different countries?

In an international exchange system, there are three possibilities: exchange of surplus organs, exchange of organs to support patients with a potentially excessive waiting time due to HLA antibodies or rare alleles, and exchange of organs to make the best possible match between donor and recipient.

A classical point system is unlikely to work, as this would imply that donor rates and donor structures as well as recipient populations between the countries are very similar. Even if donor rates vary within one country solidarity is hard to uphold. If one country exports otherwise not used organs, in other words has an organ surplus, it wants some benefits in exchange. These benefits could be better organs or a better HLA match for its recipients. However, whenever balancing points are used to equalise different donor rates or waiting times, HLA matching gets worse.

Before setting up an international organ exchange system, some preconditions have to be met; first a country that exports an organ should be rewarded with an organ of equal quality whenever possible. Furthermore, the standards of organ procurement have to be accepted between the partners and the exchange of organs across borders has to be legally possible. In the interest of fairness, full donor reporting has to be achieved and managed.

Primary allocation should be performed within the countries. One could simply define the minimal requirements regarding organ quality and HLA matching for every recipient. Organ quality could be defined by age, cause of death and/or certain diseases or even multidimensional scoring system such as kidney donor risk index/kidney donor profile index (KDRI/KDPI).

The benefit of international organ exchange is most pronounced in highly sensitized patients or those with rare alleles. If patients were likely to wait extremely long due to their rare HLA type or presensitization, they would accept any organ rather than die on dialysis.

\section{Current experiences with international exchange programs}

The major benefit from international exchange is the possibility to transplant highly sensitized patients and those with rare conditions such as rare HLA alleles. As an example of 280 hearts offered to Swiss transplant 2,8\% were accepted and transplanted for patients with rare conditions equalling $5.8 \%$ of the Swiss heart transplant activity (42). 
Other countries could markedly increase their transplant activities and donation rates by joining an international organisation such as Croatia, Hungary, and Slovenia (43).

At present there are two organizations which are based on multinational exchange: Scandiatransplant and Eurotransplant.

In Scandiatransplant, 10 centers in 4 countries are organized. In Scandiatransplant livers are exchanged between countries based on certain criteria only (HU, no recipient). The pay back is effective immediately based on predefined criteria of quality (age, BMI, days on ICU, ALT/AST level). For donor kidneys the criteria of quality are not clearly defined.

In Eurotransplant 71 centers in 8 countries are organized. In the standard exchange program kidneys are allocated by points based on number of matches, ischemia time, mismatchprobability, waiting time and country balance. This system worked very nicely until rates of donation started to differ between countries. Furthermore, while in the beginning only donor after brain death existed, now there is a high proportion of DCD which cannot be easily exchanged due to different law systems.

In this situation, countries with a high donor rate could only reasonably participate in international organ exchange if they exported organs to accumulate balance points. Waiting time and balance points accumulated and diminished HLA compatibility. The result was a shipment for the reason of organ balance without benefit for the recipient.

\section{Exchange of surplus organs}

It is a routine between many big allocation organisations to ask their respective partner organisations whether they would have a recipient for an organ that could not be allocated within the primary organisation. In general, this is a rare condition and managed interindividually.

\section{Exchange of organs to support patients with a potentially excessive waiting time} In order to enlarge the donor pool for potential recipients and take advantage of the different distrubution of certain HLA alles between coutries, the easiest mode of operation would be the acceptance of common criteria for such potential recipients. Once the recipient has been accepted by the partnering organisations, he would be put on the list of every allocation organisation and within these organisations allocated as if he would be a member of this organisation. Once an organ has been transplanted, a pay-back would be mandatory.

As EUROSTAM has proven, this approach would be feasible between organisations and countries based on mutual trust (39).

\section{Exchange of organs to achieve the best possible match}

The legal systems vary between countries, so are donor rates, age distribution of potential recipients and access to the waiting list. Therefore, any international exchange should not be hampered by different allocation schemes within the contributing countries.

If HLA matching would be the only criterion for allocation, a cross border exchange would guarantee the optimal match. However, in such a system the differences in donor rates and 
number of potential recipients will lead to an imbalance in organ distribution. Thus, one country would export almost all organs whereas another would import almost all organs. In Eurotransplant even the inclusion of waiting time and a local bonus were not able to ameliorate the large differences in donor rates and access to the waiting list between countries. Therefore, in order to achieve a balance between countries, every country has to define, in which case organs are considered for international exchange. In other words, what are the minimal matching requirements for keeping an organ in the country.

This could be achieved in a hierarchical approach. For example, if a country wants to have a program in which old organs are allocated to old recipients only, another country could allocate any organ to old recipients, but only young organs to young recipients. As long as the organ, which is allocated cross border, has the same quality as the one, which is given back, such a scheme would have no disadvantages. Thus, if an organ in a certain country cannot be allocated to a recipient, as the criteria of such an organ do not fit to anyone on the waiting list, this organ would be allocated internationally. The accepting country would then have the obligation of immediate pay-back.

One could take this approach even further. If an organ for reasons, the countries have to define themselves, cannot usefully be transplanted into a recipient in the harvesting country, this could be offered internationally with the obligation to give one back.

Such an approach would also allow countries with an extremely low donor rate to participate in international exchanges. In these countries almost all organs can be allocated locally. However, should this not be the case, an organ could be exported. In a second step, the country/organisation, which accepted the organ, would have the obligation to pay back this organ. The sending country could then define, that it would accept this organ only for patients with high PRA or rare HLA antigens.

Within a country, a hierarchical approach means that the decision which organ is suitable for a certain recipient is one of physician and patient. Patient and physician define matching criteria (full-house, only HLA DR, HLA DR + HLA A/B/C or just one HLA in case of homozygosity) as well as the suitable organ quality (young donor, marginal donor, elderly donor, Hepatitis C, brain dead only, death after circulatory death, donor's eGFR) (table 2).

In case an organ is offered which matches the criteria of more than one potential recipient, the one with the longest waiting time would get the offer.

\section{Conclusion}

In summary, no allocation rules will fit every country around the world. A possible approach to exchange organs between countries is a hierarchical system based on organs of similar quality.

1. Allocation to recipients with special requirements (High-Urgency, highly sensitized), 2. Identification of a within the region/country, 3. In case no suitable recipient can be identified, offer countrywide or between countries, 4 . For every organ transferred to another country, there must be an obligation to give one back. 


\section{Bullet points:}

Exchange should focus on pre-sensitized recipients and organs which cannot reasonably transplanted in home country

A hierarchical approach is preferable to a point based one

International exchange should be based on pay-back

\section{Acknowledgements:}

This work would not have been possible without the extremely valuable contributions of Alexander R. Rosenkranz Division of Nephrology, Department of Internal Medicine, Medical University of Graz,Umberto Maggiore UO Nefrologia (Azienda OspedalieraUniversitaria Parma), Parma, Italy, Martina Koch Viszerale Organtransplantation /Transplantationsimmunologie, Klinik für Allgemein-, Viszeral- und Transplantationschirurgie, Langenbeckstr. 1, 55131 Mainz, Burkhard Tönshoff Department of Pediatrics I, University Children's Hospital Heidelberg,Germany, Nikolina Basic-Jukic Department of nephrology, arterial hypertension, dialysis and transplantation, University hospital centre Zagreb and Faculty of medicine, University of Zagreb, Zagreb, Croatia, Christian Hugo Division of Nephrology, Medical Clinic III, University Hospital Dresden, Dresden, Germany, Miha Arnol University Medical Centre Ljubljana, Department of Nephrology, Centre for Kidney Transplantation, Ljubljana, Slovenia, Laszlo Wagner Department of Transplantation and Surgery, Semmelweis University, Budapest, Hungary, Frans Claas Department of Immunohematology and Blood Transfusion, Leiden University Medical Center, Leiden, The Netherlands, Maarten HL Christiaans Department of Internal Medicine, Division of Nephrology, Maastricht University Medical Centre, Maastricht, The Netherlands, Claude Braun Hôpitaux Robert Schuman, Luxembourg, Luxembourg and Ingeborg Hauser Department of Nephrology, University Clinic Frankfurt, Frankfurt, Germany

\section{Financial support and sponsorship}

Nothing to declare

\section{Conflict of interests}

The authors have nothing to declare 
Table 1. Factors used for allocation in different countries

\begin{tabular}{|c|c|c|c|c|c|c|c|c|c|c|}
\hline & Waiting Time & Age & $\begin{array}{c}\text { Prior Living } \\
\text { Donor }\end{array}$ & HLA Match & PRA \% & Renal Urgency & Combined Tx & Pediatric Prior. & $Y t Y$ & OtO \\
\hline Australia & + & & & + & + & & + & + & & \\
\hline Brazil & + & & & + & & + & & + & & \\
\hline Canada & + & + & & + & + & + & + & + & + & \\
\hline Chile & + & + & & + & + & + & & + & & \\
\hline Eurotransplant** & + & + & & + & + & + & + & + & & + \\
\hline France & + & + & & + & + & + & & & + & \\
\hline Hong Kong & + & + & & + & & & & + & & \\
\hline India & + & + & + & & + & + & & & & \\
\hline Israel & + & + & & + & + & & & & & \\
\hline Italy & + & + & & + & + & + & + & + & & \\
\hline Mexico & + & + & & + & & & & & & \\
\hline New Zealand & + & & & + & & & + & & & \\
\hline Saudi Arabia & + & + & + & + & + & + & & & + & + \\
\hline ScandiaTransplant & & + & & + & + & + & & + & + & \\
\hline Singapore & + & $+* * * *$ & & + & $+* * *$ & & & & & \\
\hline South Africa & + & + & & + & + & + & & & & \\
\hline Spain & + & + & & + & + & & + & + & & \\
\hline Turkey & + & + & & + & & & & + & & \\
\hline United Kingdom & + & + & & + & + & & & + & + & + \\
\hline USA* & + & + & + & + & + & + & & + & & \\
\hline $\begin{array}{l}\text { YtY: Young to Young, } \\
* \text { - USA has a specific sy } \\
* * \text { - Eurotransplant Sen } \\
* * * \text { - Elevated PRA dec } \\
* * * *-\text { No transplantat }\end{array}$ & $\begin{array}{l}\text { tO: Old to Old } \\
\text { stem using KDF } \\
\text { or Programme } \\
\text { eases total poi } \\
\text { on over } 60 \text { yea }\end{array}$ & $\begin{array}{l}\text { I and EP } \\
\text { allocate } \\
\text { nt for th } \\
\text { s of age }\end{array}$ & $\begin{array}{l}\text { S for allocat } \\
\text { kidneys age } \\
\text { allocation }\end{array}$ & >65 years $t$ & ally pa & aged $>65$ yrs & & & & \\
\hline
\end{tabular}


Table 2: Possible Scheme of Allocation as planned in Eurotransplant

tier 1

Highly sensitized recipient
priority for pay back
sub-region
region
country
Eurotransplant
Full-house match
priority for pay back
sub-region
region
country
Eurotransplant
2 HLA-DR matches +2 matches HLA
A/B
priority for pay back
sub-region
region
country
Eurotransplant
2 HLA DR matches
priority for pay back
sub-region
region
country
Eurotransplant

Organs are categorized into groups according to age, kidney function and blood group. The organs are then allocated in the order of tiers. Within each tier organs are first allocated to countries which have exported organs, then locally, per sub-region, per region, per country and thereafter across the whole of Eurotransplant. 


\section{References}

1 Wolfe RA, Ashby VB, Milford EL et al. Comparison of mortality in all patients on dialysis, patients ondialysis awaiting transplantation, and recipients of a first cadaveric transplant. $N$ Engl J Med 1999;341:1725-30.

2 Abecassis M, Bartlett ST, Collins AJ et al. Kidney transplantation as primary therapy for end-stage renal disease: a National Kidney Foundation/Kidney Disease Outcomes Quality Initiative (NKF/KDOQITM) conference. Clin J Am Soc Nephrol 2008;3:471-80

3 Langer RM, Cohen B, Rahmel A. History of eurotransplant. Transplant Proc. 2012;44:2130-1

4 Israni AK, Salkowski N, Gustafson S et al. New national allocation policy for deceased donor kidneys in the United States and possible effect on patient outcomes. J Am Soc Nephrol. 2014;25:1842-8

5 Haller M, Gutjahr G, Kramar R, et al. Cost-effectiveness analysis of renal replacement therapy in Austria. Nephrol Dial Transplant. 2011;26:2988-95

6 CENSIS Un'analisi empirica dei consumi sanitari e dei costi dei trapiantati di rene in Italia. Dec 17 2013; available at www.censis.it

7 Burns A, Davenport A. Maximum conservative management for patients with chronic disease stage 5. Hemodial Int. 2010; Suppl 1:S32-7.

8 Goodkin DA, Bragg-Gresham JL, Koenig KG, et al. Association of comorbid conditions and mortality in hemodialysis patients in Europe, Japan, and the United States: the Dialysis Outcomes and Practice Patterns Study (DOPPS) J Am Soc Nephrol. 2003;14:3270-7

9 Meier-Kriesche HU, Kaplan B. Waiting time on dialysis as the strongest modifiable risk factor for renal transplant outcomes: a paired donor kidney analysis. Transplantation. 2002;741377-81

10 Lynch RJ, Zhang R, Patzer RE, Larsen CP, Adams AB. First-year waitlist hospitalization and subsequent waitlist and transplant outcome. Am J Transplant. 2017;17:1031-41

11 Wong G, Chua S, Chadban SJ, Clayton P, Pilmore H, Hughes PD, Ferrai P, Lim WH. Waiting Time Between Failure of First Graft and Second Kidney Transplant and Graft and Patient Survival. Transplantation. 2016;100:1767-75

12 Scalea JR, Barth RN, Munivenkatappa R et al. Shorter waitlist times and improved graft survivals are observed in patients who accept Hepatitis C virus+ renal allografts. Transplantation. 2015;99:1192-6.

20 Carrier M, Lize JF; Quebec-Transplant Programs. Impact of expanded criteria donors on outcomes of recipients after kidney transplantation. Transplant Proc. 2012;44:2227-30

14 Debout A, Foucher Y, Treben-Launay K, et al. Each additional hour of cold ischemia time significantly increase the risk of graft failure and mortality following renal transplantation. Kidney Int. 2015;87:343-9

15 Johnson RJ, Fuggle SV, O’Neill J et al. Kidney Advisory Group of NHS Blood and Transplant. Factors influencing outcome after deceased heart beating donor kidney transplantation in the United Kingdom: an evidence base for a new national kidney allocation policy. Transplantation. 2010;89379-86

16 Bahde R, Vowinkel T, Unser J et al. Prognostic factors for kidney allograft survival in the Eurotransplant Senior Program. Ann Transplant. 2014;19:201-9

17 Molnar MZ, Streja E, Kovesdy CP et al. Age and the associations of living donor and expanded criteria donor kidneys with kidney transplant outcomes. Am J Kidney Dis. 2012;59:841-8

18 Knoll GA. Kidney transplantation in the older adult. Am J Kidney Dis. 2013;61:790-7

19 Wehmeier C, Georgalis A, Hirt-Minkowski P et al. 2222 kidney transplantations at the University Hospital Basel: a story of success and new challenges. Swiss Med Wkly. 2016:146:w14317

20 http://www.trapianti.salute.gov.it/cnt

21 Matesanz R, Domínguez-Gil B, Coll E, Mahíllo B, Marazuela R. How Spain Reached 40 Deceased Organ Donors per Million Population. Am J Transplant 2017;17:1447-54**

This article summarises the activities of the most successful donor campaign

22 Annual report $2018 \mathrm{ET}^{* *}$

Illustrates the activities of the largest international organ exchange organisation

23 https://optn.transplant.hrsa.gov 
24 Siddiqi N, McBride MA, Hariharan S. Similar risk profiles for post-transplant renal dysfunction and longterm graft failure: UNOS/OPTN database analysis. Kidney Int. 2004;651906-13

25 Lim WH, Chadban SJ, Clayton P et al. Human leukocyte antigen mismatches associated with increased risk of rejection, graft failure, and death independent of initial immnosuppression in renal transplant recipients. Clin Transplant. 2012;26:E428-37

26 Opelz G, Döhler B. Association of HLA mismatch with death with a functioning graft after kidney transplantation: a collaborative transplant study report. Am J Transplant. 2012;12:3031-8

27 Foster BJ, Dahhou M, Zhang X, et al. Impact of HLA mismatch at first kidney transplant on lifetime with graft function in young recipients. Am J Transplant. 2014;14:876-85

28 Doxiadis II, de Fijter JW, Mallat MJ, et al. Simpler and equitable allocation of kidneys from postmortem donors primarily based on full HLA-DR compatibility Transplantation. 2007;83:1207-13*

This article proves the concept and feasibility of HLA-DR matching

29 Johnson RJ, Fuggle SV, Mumford L et al. Kidney Advisory Group of NHS Blood and Transplant. A New UK 2006 National Kidney Allocation Scheme for deceased heart-beating donor kidneys. Transplantation. 2010;89:387-94

30 Lim WH, Chapman JR, Wong G. Peak panel reactive antibody, cancer, graft, and patient outcomes in kidney transplant recipients. Transplantation. 2015;99:1043-50

31 Johnson C, Ellis T Reply to "Allocation Based on Virtual Crossmatch Alone: Not Yet Ready for Primetime". Am J Transplant 2016; 16: 3578-9

32 Heidt S, Haasnoot GW, van Rood JJ, Witvliet MD, Claas FHJ Kidney allocation based on proven acceptable antigens results in superior graft survival in highly sensitized patients. Kidney Int. 2017. pii: S0085-2538(17)30549-5*

This article describes the concept and results of the first program for highly sensitized recipients

33 http://cordis.europa.eu/result/rcn/181463_it.html

34 Maggiore U Oberbauer R, Pascual J et al. Strategies to increase the donor pool and access to kidney transplantation: an international perspective. Nephrol Dial Transplant 2015; 30:217-22

35 Ekser B, Mangus RS, Fridell W et al.A novel approach in combined liver and kidney transplantation with long-term outcomes. Ann Surg. 2017 265:1000-1008

36 Reich H, Dimbil S, Levine R et al. Dual-organ transplantation in older recipients: outcomes after heartkidney transplant versus isolated heart transplant in patients aged $\geq 65$ years. Interact Cardiovasc Thorac Surg. 2019; 28:45-51

37 Costa MG, Garcia VD, Leirias MM et al. Urgency priority in kidney transplantation in Rio Grande do Sul. Transplant Proc. 2007;39:381-2

38 Celebi ZK, Akturk S, Erdogmus S et al. Urgency Priority in Kidney Transplantation: Experience in Turkey. Transplant Proc. 2015;47:1269-72

39 Assfalg V, Hüser N, Heemann U et al. High urgency kidney transplantation in Eurotransplant Kidney Allocation System (ETKAS): success or waste of organs? The Eurotransplant 15-year all center survey. Nephrol Dial Transplant 2016;31:1515-22*This article highlights the benefits and sortcomings of HU programs

40 http://www.odt.nhs.uk/pdf/kidney_allocation_policy.pdf

41 https://www.eurotransplant.org/cms/index.php?page=et_manual

42 Schneider S, Schreiner P, Weiss J, Immer FF. Assessing the potential of international organ exchange-the Swiss experience. Eur J Cardiothorac Surg. 2011;40(6):1368-73)

43 Langer R. The first five years of Hungary's EUROTRANSPLANT membership. Orv Hetil. 2018;159(42):1695-1699 